\title{
pH-Responsive Nano-Self-Assemblies of the Anticancer Drug 2-Hydroxyoleic Acid
}

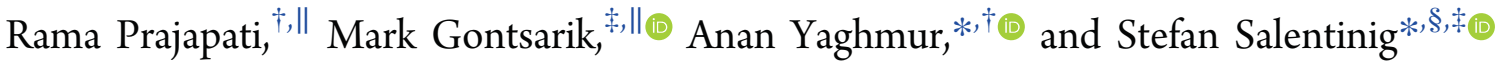 \\ ${ }^{\dagger}$ Department of Pharmacy, Faculty of Health and Medical Sciences, University of Copenhagen, Universitetsparken 2, DK-2100 \\ Copenhagen $\varnothing$, Denmark \\ ${ }^{\ddagger}$ Laboratory for Biointerfaces, Empa, Swiss Federal Laboratories for Materials Science and Technology, Lerchenfeldstrasse 5, 9014 \\ St. Gallen, Switzerland \\ ${ }^{\S}$ Department of Chemistry, University of Fribourg, Chemin du Musée 9, 1700 Fribourg, Switzerland
}

\section{Supporting Information}

\begin{abstract}
H}$-responsive lipid nanocarriers have the potential to selectively target the acidic extracellular $\mathrm{pH}$ environment of cancer tissues and may further improve the efficacy of chemotherapeutics by minimizing their toxic sideeffects. Here, we present the design and characterization of $\mathrm{pH}$-sensitive nano-self-assemblies of the poorly water-soluble anticancer drug 2-hydroxyoleic acid (2OHOA) with glycerol monooleate (GMO). pH-triggered nanostructural transformations from 2OHOA/GMO nanoparticles with an internal inverse hexagonal structure (hexosomes) at $\mathrm{pH}$ around 2.0-3.0, via nanocarriers with an internal inverse bicontinuous cubic structure (cubosomes) at $\mathrm{pH} 2.0-4.5$, to vesicles at $\mathrm{pH} 4.5-7.4$ were observed with synchrotron small-

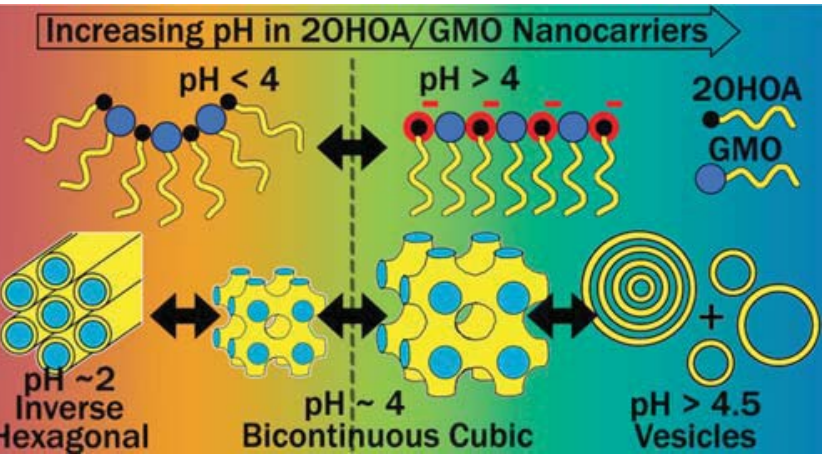

angle X-ray scattering, and cryogenic transmission electron microscopy. $\zeta$-potential measurements highlight that the $\mathrm{pH}$-driven deprotonation of the carboxylic group of $2 \mathrm{OHOA}$, and the resulting charge-repulsions at the lipid-water interface account for these nanostructural alterations. The study provides detailed insight into the $\mathrm{pH}$-dependent self-assembly of $2 \mathrm{OHOA}$ with GMO in excess buffer at physiologically relevant $\mathrm{pH}$ values, and discusses the effects of $\mathrm{pH}$ alterations on modulating their nanostructure. The results may guide the further development of $\mathrm{pH}$-responsive anticancer nanocarriers for the targeted delivery of chemotherapeutics to the local microenvironment of tumor cells.
\end{abstract}

\section{INTRODUCTION}

Cancer is one of the major public health problems worldwide. $^{1-3}$ Along with the current cancer therapies, including surgical intervention and radiation therapy, chemotherapy is characterized as the least invasive cancer treatment approach with a clinical success in tumor shrinking and cancer relapse reduction. ${ }^{4-6}$ However, chemotherapy is often associated with side-effects caused by the off-site toxicity due to the lack of drug specificity. ${ }^{5}$ Hence, the design of novel and more efficient cancer nanomedicines that can selectively target the tumor sites, avoiding off-target side-effects, may improve current therapies and also patients' quality of life. ${ }^{5,7-10}$ Among different suggested nanomedicines, $\mathrm{pH}$-triggered chemotherapeutic delivery systems may be of interest in cancer treatment, as the extracellular microenvironment of tumor cells with $\mathrm{pH}$ of around 5.5-6.5 is more acidic than that of healthy cells with $\mathrm{pH}$ of 7.4. ${ }^{10-12}$ The occurrence of such acidic local tumor microenvironment is attributed to an increased glycolysis rate, a higher production level of lactic acid, and an insufficient vasculature for the removal of acidic byproducts. ${ }^{11,13-18}$

This study describes the formation and characterization of $\mathrm{pH}$-responsive self-assembled nanocarriers based on glycerol monooleate (GMO), and the anticancer drug 2-hydroxyoleic acid (2OHOA). The molecular structures of both lipids are presented in Figure 1. The poorly water-soluble anticancer drug 2OHOA is a derivative of oleic acid (OA) with an additional hydroxyl group on the $\alpha$-carbon of the fatty acid backbone. ${ }^{19}$ It is a potent anticancer agent that induces cell

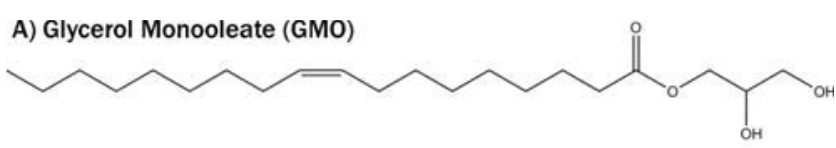

B) 2-Hydroxyoleic Acid (2OHOA)

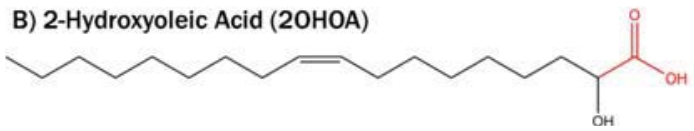

Figure 1. Molecular structures of the lipids: (A) glycerol monooleate (GMO) and (B) 2-hydroxyoleic acid (2OHOA). The carboxylic group of $2 \mathrm{OHOA}$ is highlighted in red color in panel $\mathrm{B}$. 
cycle arrest and apoptosis, cellular differentiation, and autophagy in a wide range of human cancer cells, including leukemia, glioma, lung, colon, and breast cancer cells. ${ }^{19-27}$

GMO-based nanocarriers have been previously used for enhancing the solubilization of poorly water-soluble therapeutic agents. ${ }^{28-35}$ Among these nanocarriers, dispersions of inverse bicontinuous cubic, hexagonal, and micellar cubic structures (cubosomes, hexosomes, and micellar cubosomes, respectively), emulsified microemulsions, vesicles, and sponge phases are gaining increasing attention for their drug delivery applications. The biological relevance and the relatively large extent of the internal lipid-water interfacial area render these colloidal nanosystems attractive for delivering hydrophilic, hydrophobic, and amphiphilic drugs and peptide molecules. $^{36-48}$ For targeted delivery applications, they can be surface nanoengineered to respond to an external stimulus, such as $\mathrm{pH}$, for triggering the release of therapeutic molecules or enhancing the interactions with cells on demand, owing to structural alterations in these nanocarriers. ${ }^{33,49-55}$ Despite the attractiveness of cubosomes, hexosomes, and related nonlamellar liquid crystalline nanoparticles in the development of injectable drug nanocarriers, there is still to date no FDAapproved formulation based on these nanostructured emulsions. $^{56,57}$ Nevertheless, recent in vivo studies showed that these nanoparticles are promising candidates in the development of nanocarriers for anticancer drug delivery. ${ }^{58,59}$

The major goal of this study is to investigate the composition and $\mathrm{pH}$ dependence of 2OHOA/GMO nanoself-assemblies and gain further insight into their $\mathrm{pH}$-triggered structural, morphological, and size alterations by using synchrotron small-angle X-ray scattering (SAXS), cryogenic transmission electron microscopy (cryo-TEM), and $\zeta$-potential measurements. An improved understanding of the structural mechanism involved in these $\mathrm{pH}$-triggered transitions is an important keystone toward the development of $\mathrm{pH}$-responsive anticancer nanomedicines. Based on the presented experimental findings, the $\mathrm{pH}$-induced phase transformations in the interiors of 2OHOA/GMO nanoparticles are most likely attributed to the protonation/deprotonation of the carboxylic group of 2OHOA at the lipid-water interface. The presented results shed light on the molecular interactions during the selfassembly of a cell membrane active anticancer agent with a biologically relevant lipid in excess buffer and could potentially be applied for the future design of $\mathrm{pH}$-guided nanocarriers for tumor-targeted delivery of anticancer drugs.

\section{EXPERIMENTAL SECTION}

Preparation of 2OHOA/GMO Nanodispersions. Sodium salt of 2-hydroxyoleic acid (2OHOA; Avanti polar lipids, Alabama, U.S.A.) and glycerol monooleate (GMO; $\geq 90 \%$ purity, Riken Vitamin co., Ltd. company, Japan) were weighed out in glass vials at $3 / 7$ and $1 / 12$ OHOA/GMO mass ratios and dispersed in $149 \mathrm{mM}$ Dulbecco's Phosphate-buffered saline (PBS) at pH 7.4 (Sigma-Aldrich, Poole, UK), containing $2 \mathrm{mg} / \mathrm{mL}$ Pluronic F127 (gift from BASF SE, Ludwigshafen, Germany), to achieve a total lipid concentration of 20 $\mathrm{mg} / \mathrm{mL}$. These raw lipid emulsions were then homogenized by means of ultrasonication (Ultrasonic Processor Qsonica 500, Qsonica LLC, Newtown, CT, U.S.A.) for $4 \mathrm{~min}$ in pulse mode ( $3 \mathrm{~s}$ pulse, $5 \mathrm{~s}$ break) at $27 \%$ of its maximum power $(500 \mathrm{~W})$. The $\mathrm{pH}$ of the prepared nanodispersions was then adjusted with $1 \mathrm{M} \mathrm{NaOH}(\geq 99 \%$ purity, Carl Roth $\mathrm{GmbH}$, Karlsruhe, Germany) or $1 \mathrm{M} \mathrm{HCl}$ prepared from $37 \% \mathrm{HCl}$ stock solution (ACS reagent grade, Sigma-Alrdich, Buchs, Switzerland) and allowed to equilibrate at $25^{\circ} \mathrm{C}$ for at least $1 \mathrm{~h}$ before carrying out the planned experiments. In this study, the $\mathrm{pH}$ of the continuous aqueous medium of the dispersion with $1 / 1$ 2OHOA/ GMO mass ratio was first gradually decreased from 7.4 to 2.0 and then increased back to 5.0, while taking out samples at different selected $\mathrm{pH}$ points for SAXS structural characterization. This $\mathrm{pH}$ cycle was applied to study whether the structural alterations associated with changing $\mathrm{pH}$ can be reversed. Double distilled water was used for the preparation of all samples. All ingredients were used without further purification.

Synchrotron Small-Angle X-ray Scattering (SAXS). SAXS patterns were recorded at the Austrian SAXS beamline at the synchrotron light source ELETTRA (Trieste, Italy). An X-ray beam with a wavelength of $1.54 \AA$ ( $8 \mathrm{keV})$ was used, with a sample-todetector distance of $1314 \mathrm{~mm}$, covering a $q$-range from about 0.18 to $5.00 \mathrm{~nm}^{-1}$, where $q$ is the length of the scattering vector, defined by $q$ $=4 \pi / \lambda \sin (\theta / 2), \lambda$ being the wavelength, and $\theta$ the scattering angle. The samples were sealed in thin-walled quartz capillaries and five frames with an exposure time of $20 \mathrm{~s}$ per frame were collected and averaged at room temperature. The 2D SAXS patterns were acquired using a Pilatus3 $1 \mathrm{M}$ detector (Dectris Ltd., Baden, Switzerland; active area $169 \times 179 \mathrm{~mm}^{2}$ with a pixel size of $172 \times 172 \mu \mathrm{m}^{2}$ ), integrated into one-dimensional (1-D) scattering function $I(q)$ using Fit $2 D^{60}$ (European Synchrotron Radiation Facility, Grenoble, France) and then analyzed with IGOR pro (Wavemetrics, Inc., Lake Oswego, U.S.A.). The scattering curves were plotted as a function of intensity, $I(q)$, averaged over the five repeated exposures versus $q$. The scattering from PBS was subtracted as background from all measurements before further data analysis. The calculation of the lattice parameters of the corresponding lyotropic nonlamellar liquid crystalline phases is described in SI (eqs S2-S4).

Cryogenic-Transmission Electron Microscopy (cryo-TEM). The morphological characterization of F127-stabilized 2OHOA/ GMO nanoparticles was done in their vitrified states by cryo-TEM imaging. Briefly, lacey carbon 300 mesh copper grid (Ted Pella Inc., CA, U.S.A.) enforced by a silicon monoxide coating was subjected to glow discharge treatment and treated with $3 \mu \mathrm{L}$ of the sample. The excess sample on the grid was then blotted with filter paper at a blotting time of $3.5 \mathrm{~s}$, a blotting force 0 , temperature $25^{\circ} \mathrm{C}$, and $100 \%$ humidity (FEI Vitrobot IV, Holland) and was rapidly plunged into liquid nitrogen-cooled ethane close to its melting point $\left(-174^{\circ} \mathrm{C}\right)$. A Gatan 626 cryo-holder (Gatan, U.K.) was used to transfer the plunged grid with the samples in a frozen glassy state into the Tecnai G2 20 transmission electron microscope (FEI, Holland). The images were digitally recorded at a voltage of $200 \mathrm{kV}$, under low-dose conditions $\left(\sim 5 \mathrm{e} / \AA^{2} \mathrm{~s}\right)$, and at a magnification of $\times 29000$.

$\zeta$-Potential Measurements. The $\zeta$-potential of the 2OHOA/ GMO nanodispersions was measured at different $\mathrm{pH}$ using Zetasizer Nano ZS equipped with a $633 \mathrm{~nm}$ laser (Malvern Instruments, Worcestershire, U.K.). Prior to measurements at $25{ }^{\circ} \mathrm{C}$ and $173^{\circ}$ scattering angle, the samples were diluted $1 / 100$ in PBS and their $\mathrm{pH}$ was readjusted. $\zeta$-potential was calculated by Zetasizer Software 7.11 (Malvern Instruments) using Smoluchowski equation: ${ }^{61}$

$$
\mu_{\mathrm{e}}=\frac{\varepsilon_{\mathrm{r}} \varepsilon_{0} \zeta}{\eta}
$$

where $\mu_{\mathrm{e}}$ is the electrophoretic mobility, $\varepsilon_{\mathrm{r}}$ is the dielectric constant of water, $\varepsilon_{\mathrm{o}}$ is the permittivity of vacuum, and $\eta$ is the viscosity of the solvent (water). The measurements were done in triplicate and averaged. Estimations of 2OHOA's $\mathrm{p}_{\mathrm{a}}^{\mathrm{app}}$ from the $\zeta$-potential values are described in the SI.

\section{RESULTS AND DISCUSSION}

The GMO dispersed in water forms cubosomes with an internal bicontinuous cubic phase of the $P n 3 m$ or $\operatorname{Im} 3 m$ type symmetry depending on the F127 stabilizer concentration. ${ }^{62,63}$ The integration of the anticancer drug 2OHOA into this dispersion led to composition- and $\mathrm{pH}$-dependent nanostructural transformations in the nanocarriers. Figure 2 demonstrates the $\mathrm{pH}$-dependent self-assembly of the binary 


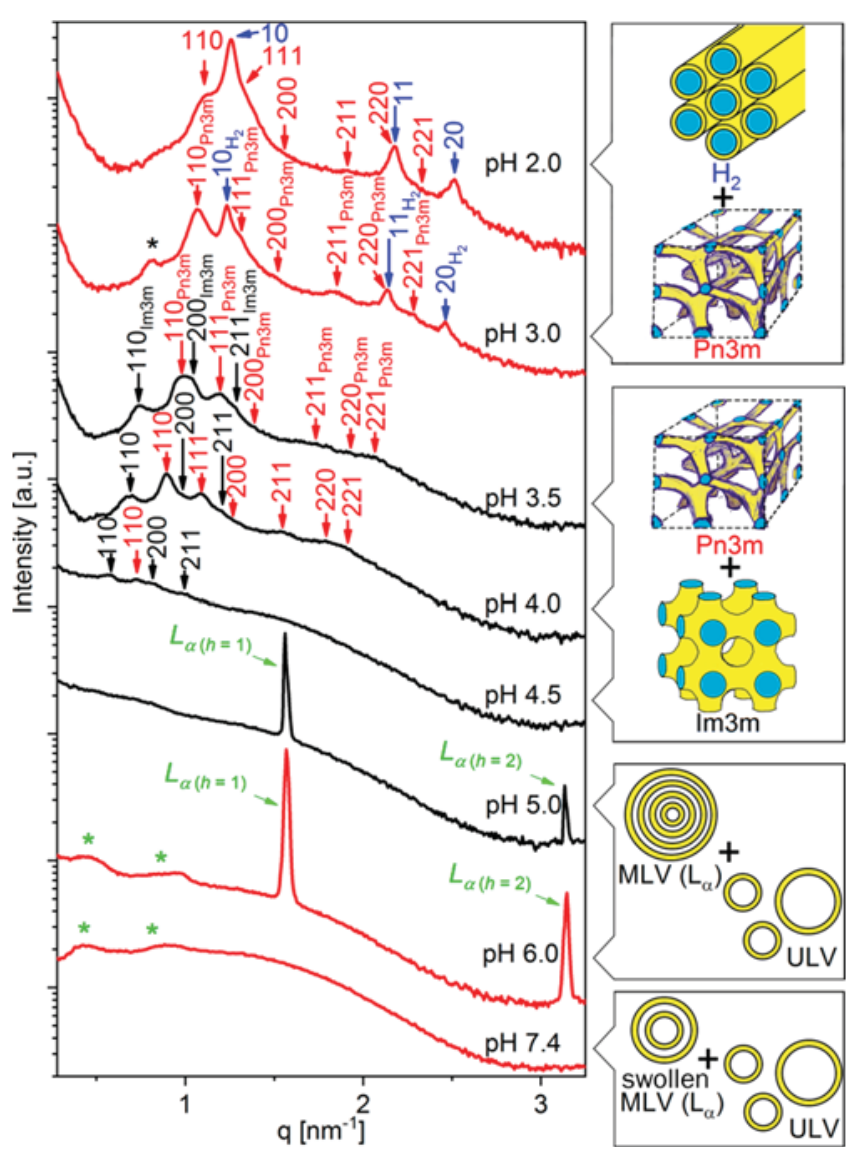

Figure 2. SAXS patterns for the F127-stabilized nano-self-assemblies prepared at $2 \mathrm{OHOA} / \mathrm{GMO}$ mass ratio of $1 / 1$ as the $\mathrm{pH}$ of the dispersion was gradually decreased from 7.4 to 2.0 (red curves) and then increased back to 5.0 (black curves). The characteristic Bragg peaks and the corresponding Miller indices of the cubic $P_{n} 3 m$ (red), Im $3 m$ (black), and $\mathrm{H}_{2}$ (blue) phases are marked with arrows. Low intensity peak attributed to a potential coexistence of $\operatorname{Im} 3 \mathrm{~m}$ phase is marked with a black asterisk at $\mathrm{pH}$ 3.0. The first two Bragg reflections from the interlamellar distance of the MLVs are marked with $\mathrm{L}_{\alpha(\mathrm{h}=1)}$ and $\mathrm{L}_{\alpha \mathrm{h}=2)}$ (green). Broad correlation peaks most likely corresponding to weak bilayer correlations in a coexisting population of swollen MLVs are marked with green asterisks.

2OHOA/GMO self-assemblies in excess buffer at a mass ratio of $1 / 1$. The appearance of three dominating Bragg reflections in the SAXS curves at $q$ values of $1.26,2.17$, and $2.51 \mathrm{~nm}^{-1}$, corresponding to a peak spacing-ratio of $1: \sqrt{ } 3: \sqrt{ } 4$, indicate the formation of hexosomes with an inverse hexagonal $\left(\mathrm{H}_{2}\right)$ phase at $\mathrm{pH}$ 2.0. The corresponding lattice parameter, $a_{\mathrm{H}_{2}}$, is $5.8 \mathrm{~nm}$. Additional weak reflections, occurring most prominently around $q \approx 1.1 \mathrm{~nm}^{-1}$, indicate a coexisting bicontinuous cubic structure with $P n 3 m$ symmetry having a lattice parameter, $a_{P n 3 m}$, of $8.0 \mathrm{~nm}$. These results demonstrate that the 2OHOA mostly integrates into the hydrophobic domains of the self-assembled nanostructure at $\mathrm{pH}$ 2.0. This integration increases the apparent volume of the hydrophobic domains, which ultimately leads to the nanostructural transition from cubosomes to hexosomes. This observation agrees with theoretical considerations from the critical packing parameter model (see SI). The trend is also consistent with 2OHOA-induced lamellar-nonlamellar structural transitions in dielaidoylphosphatidylethanolamine dispersion. ${ }^{24,64}$
Upon increasing the $\mathrm{pH}$ from 2.0 to 3.0, the Bragg peaks of the $\mathrm{H}_{2}$ phase shifted to lower $q$ values, corresponding to an increase in $a_{\mathrm{H}_{2}}$ to $5.9 \mathrm{~nm}$ (Figure 2). In addition to the characteristic reflections of the $H_{2}$ phase, additional Bragg peaks with peak-ratios of $\sqrt{ } 2: \sqrt{ } 3: \sqrt{ } 4: \sqrt{ } 6: \sqrt{ } 8: \sqrt{ } 9$ were detected. These peaks could be indexed as the (110), (111), (200), (211), (220), and (221) reflection of the bicontinuous $P n 3 m$-type cubic structure with $a_{P n 3 m}=8.3 \mathrm{~nm}$. In this phase assignment, it should be noted that the characteristic reflection (220) of the cubic $\mathrm{Pn} 3 \mathrm{~m}$ phase at $\mathrm{pH} 2.0$ and 3.0 appears to overlap with the characteristic reflection (11) of the coexisting $\mathrm{H}_{2}$ phase. A single peak around $q \approx 0.8 \mathrm{~nm}^{-1}$ was also observed, likely resulting from traces of a coexisting $\operatorname{Im} 3 m$-type bicontinuous cubic structure. The identification of this coexisting $\operatorname{Im} 3 m$ structure was based on its gradual evolvement with a further increase in $\mathrm{pH}$. At $\mathrm{pH} 3.5$, the Bragg reflections of the $\mathrm{H}_{2}$ phase diminished and peaks indicating a coexistence of $P n 3 m$ and $I m 3 m$ phase were observed. The corresponding lattice parameters for the biphasic $P n 3 m / \operatorname{Im} 3 m$ feature were 9.1 and $12.0 \mathrm{~nm}$, respectively. Further increase in $\mathrm{pH}$ to 4.0 caused a shift in the peak positions of the Pn $3 m$ and $\operatorname{Im} 3 m$ phases to lower $q$ values, resulting in an increase in $a_{P n 3 m}$ to 9.9 $\mathrm{nm}$ and $a_{\operatorname{Im} 3 m}$ to $12.9 \mathrm{~nm}$. At $\mathrm{pH} 4.5$, the lattice parameters of $P n 3 m$ and $\operatorname{Im} 3 m$ increased to 12.3 and $15.3 \mathrm{~nm}$, respectively. The ratio of $a_{I m 3 m} / a_{P n 3 m}$ was calculated and found to be 1.32 , 1.30 , and 1.24 at $\mathrm{pH} 3.5,4.0$, and 4.5. This ratio is consistent with the reported theoretical Bonnet ratio of 1.279 for these structures $^{36,65}$ (Table S1). At pH 5.0 and 6.0, two Bragg peaks at $q$ values of about 1.56 and $3.12 \mathrm{~nm}^{-1}$ were observed, showing the formation of multilamellar vesicles (MLVs) with an interlamellar $d$-spacing of about $4.0 \mathrm{~nm}$, in agreement with the previously reported bilayer dimensions in GMO-based selfassemblies. ${ }^{66,67}$ At $\mathrm{pH}$ 7.4, the diffuse-dominating scattering patterns indicated the formation of unilamellar vesicles (ULVs). The additional broad correlation peaks in the low- $q$ region of the SAXS curves at $\mathrm{pH} 6.0$ and 7.4, with maxima around $q \approx 0.45$ and $0.90 \mathrm{~nm}^{-1}$ are most likely attributed to weak bilayer correlations in a coexisting population of swollen bi- or multilamellar vesicles with an estimated $d$ of around $\sim 14$ $\mathrm{nm}$. The formation of highly swollen lamellar structures with similar bilayer-to-bilayer spacing and low repeat numbers was also previously reported for nano-self-assemblies of GMO with the anionic phospholipid dioleoyl-phosphatidylglycerol. ${ }^{67}$

To evaluate the potential to reverse the colloidal transformations in this system, the $\mathrm{pH}$ in the sample at $1 / 1$ 2OHOA/GMO mass ratio was decreased from $\mathrm{pH} 7.4$, where vesicles were the dominating species, to $\mathrm{pH} 2.0$ where cubosomes and hexosomes dominated (Figure 2). The following $\mathrm{pH}$ increase to 5.0 in this sample led to the reformation of vesicles, demonstrating the $\mathrm{pH}$-induced transformations from nonlamellar structures to vesicles and back upon circulating the $\mathrm{pH}$ of the continuous water phase. The $\mathrm{pH}$-triggered swelling of nonlamellar liquid crystalline structures and the gradual nonlamellar-lamellar transitions have been previously reported for self-assemblies containing the analogue $\mathrm{OA}^{50,52,69,70}$ and other fatty acids. ${ }^{49,71}$

The cryo-TEM images of the 2OHOA/GMO nano-selfassemblies at $1 / 1$ mass ratio confirmed the $\mathrm{pH}$-triggered nanostructural transitions observed by SAXS. Hexosomes were observed at $\mathrm{pH} 2.0$, and internally structured nanoparticles coexisting with vesicles were found at $\mathrm{pH} 4.0$ (Figure 3A,B). At $\mathrm{pH}$ 6.0, the cryo-TEM image (Figure 3C) indicated the 


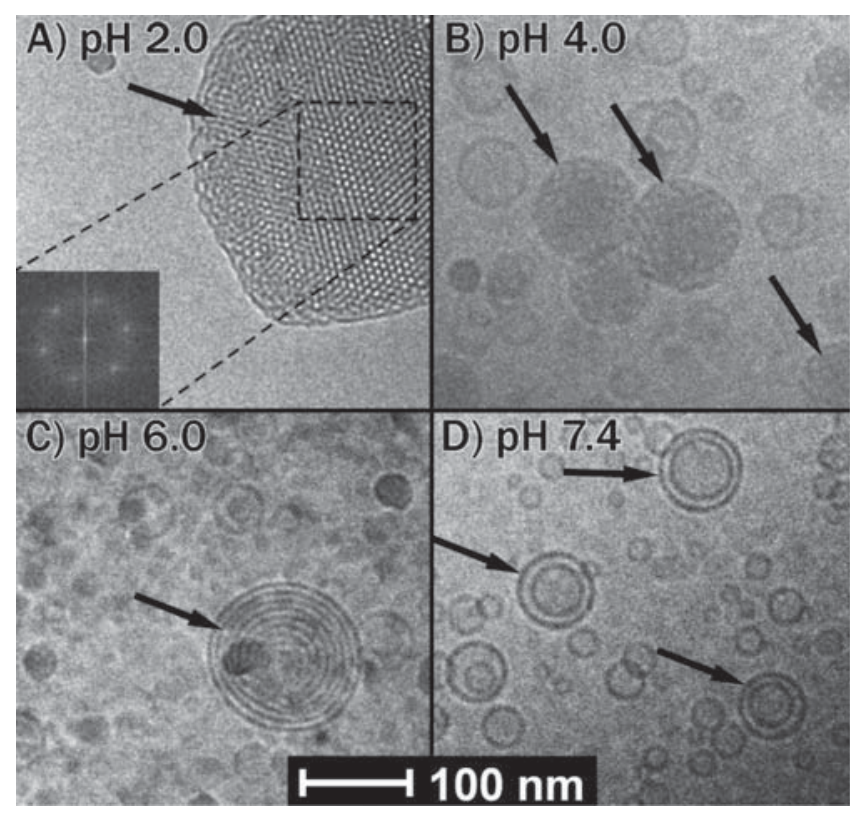

Figure 3. Representative cryo-TEM images of the nanodispersions prepared at $2 \mathrm{OHOA} / \mathrm{GMO}$ mass ratio of $1 / 1$ and the following $\mathrm{pH}$ : (A) $\mathrm{pH}$ 2.0, (B) $\mathrm{pH} 4.0$, (C) $\mathrm{pH}$ 6.0, and (D) $\mathrm{pH}$ 7.4. The insert in panel A shows the fast Fourier transformation (FFT) applied to the structured part of the image indicating the formation of hexosomes. Colloidal transitions from (A) hexosomes to (B) cubosomes, and (C) MLVs (marked with black arrows) coexisting with relatively small nano-self-assemblies were observed with increasing $\mathrm{pH}$. Small ULVs of variable sizes from around 100 to $15 \mathrm{~nm}$ in diameter were observed at $\mathrm{pH}$ 7.4, coexisting with multilamellar vesicles (marked with black arrows) with a bilayer-to-bilayer distance of around $\sim 10 \mathrm{~nm}$ (D).

presence of MLVs coexisting with smaller ULVs. The image at $\mathrm{pH} 7.4$ (Figure 3D) was dominated by ULVs mostly below $100 \mathrm{~nm}$ in diameter, coexisting with bilamellar vesicles. The bilayer-to-bilayer distance of around $10 \mathrm{~nm}$ in the cryo-TEM of multilamellar vesicles at $\mathrm{pH} 7.4$ (Figure 3D) correlates reasonably well with the estimated interlamellar distance from SAXS (Figure 2).

Figure 4 shows the SAXS patterns for the 2OHOA/GMO nano-self-assemblies at a $2 \mathrm{OHOA} / \mathrm{GMO}$ mass ratio of $3 / 7$, in the $\mathrm{pH}$ range of 2.0-7.4. At $\mathrm{pH} 2.0$, the SAXS curve shows the characteristic peak-spacing of the $\mathrm{Pn} 3 \mathrm{~m}$ type cubic phase with $a_{P n 3 m}$ of $8.39 \mathrm{~nm}$. Hence, compared to the dispersion that was prepared at a mass ratio of $1 / 1$, the $2 \mathrm{OHOA}$ content in this sample is insufficient to induce a $\mathrm{Pn} 3 m$-to- $\mathrm{H}_{2}$ phase transition even at $\mathrm{pH}$ 2.0. On increasing $\mathrm{pH}$ to 3.0 and 3.5 , the calculated lattice parameter of the cubic $P n 3 m$ phase increased to 8.6 and $9.3 \mathrm{~nm}$, respectively. However, three additional Bragg peaks at $q$ values of $0.72,1.02$, and $1.25 \mathrm{~nm}^{-1}$ with peak spacing-ratio of $\sqrt{ } 2: \sqrt{ } 4: \sqrt{ } 6$ were observed at $\mathrm{pH} 3.5$, which can be attributed to a coexisting $\operatorname{Im} 3 m$ phase with $a_{\operatorname{Im} 3 m}$ of $12.3 \mathrm{~nm}$. The calculated ratio between the lattice parameters of $\operatorname{Im} 3 m$ and $P n 3 m$ phases was 1.33 and fairly consistent with the theoretical Bonnet ratio (Table S1). The SAXS curve at $\mathrm{pH} 4.0$ was dominated by the $\operatorname{Im} 3 m$ phase with $a_{\operatorname{Im} 3 m}$ of about $14.6 \mathrm{~nm}$, as the three $\operatorname{Im} 3 m$ peaks were shifted to lower $q$ values. Further increase in $\mathrm{pH}$ to 4.5-7.4 led to the loss of the highly ordered nonlamellar structures and the appearance of diffusive SAXS curves, indicating a transformation to vesicles. The correlation peaks observed in the low- $q$ region of curves at $\mathrm{pH} 6.0$ and 7.4, with their maxima around 0.48 and $0.96 \mathrm{~nm}^{-1}$, may correspond

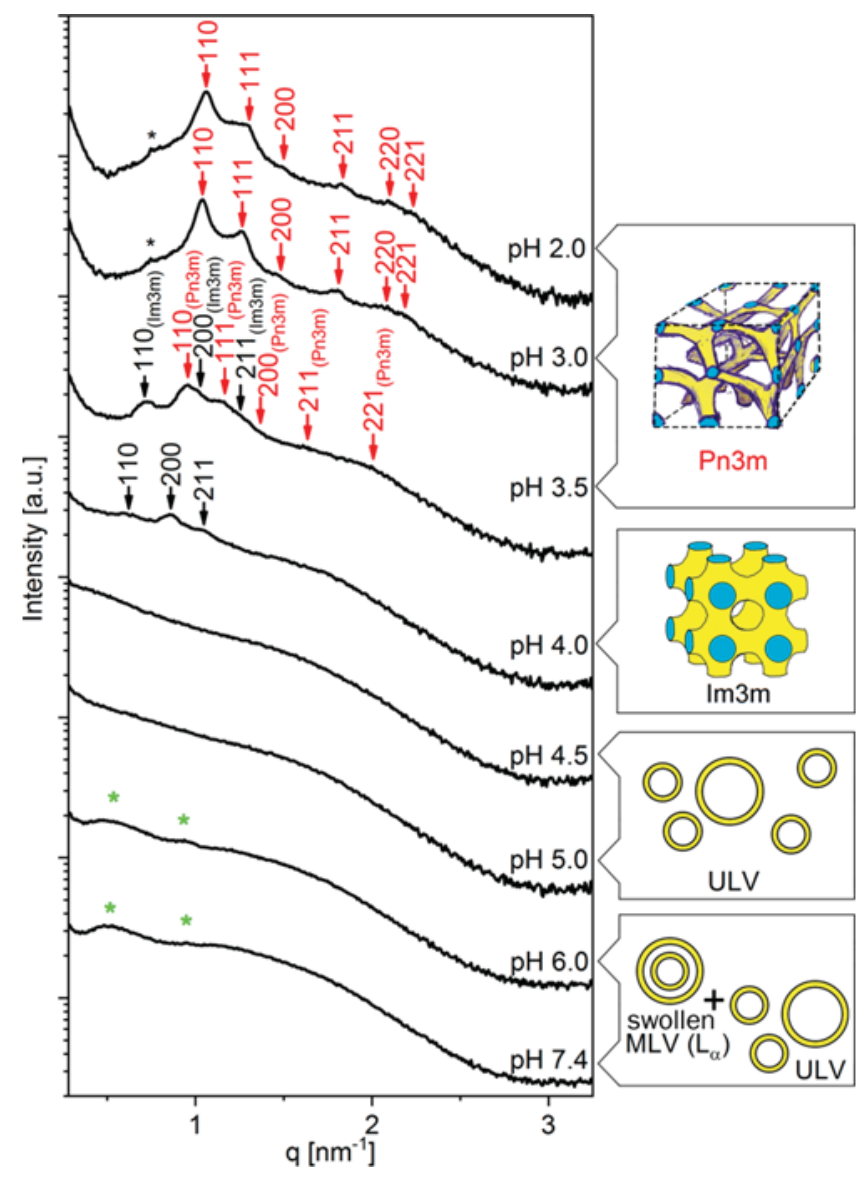

Figure 4. SAXS patterns of the F127-stabilized nanoself-assemblies prepared at 2OHOA/GMO mass ratio of $3 / 7$ and $\mathrm{pH}$ in the range of 2.0-7.4. The Bragg peaks of the cubic $P n 3 m$ and $\operatorname{Im} 3 m$ phases and their corresponding Miller indices are indicated with red and black arrows, respectively. Low intensity peaks potentially from a coexisting $\operatorname{Im} 3 \mathrm{~m}$ phase are marked with black asterisks at $\mathrm{pH}$ of 2.0 and 3.0. Broad correlation peaks, most likely corresponding to the weak bilayer correlations in coexisting swollen MLVs, are marked with green asterisks at $\mathrm{pH} 6.0$ and 7.4.

to the weak bilayer correlations of a coexisting population of highly swollen MLVs with an estimated $d$ of around $13 \mathrm{~nm}$ in agreement with the discussions above.

The structural transformations of the 2OHOA/GMO nanoself-assemblies upon increasing $\mathrm{pH}$ from 2.0 to 7.4 are attributed to the charge repulsions between the gradually deprotonating carboxylic groups of $2 \mathrm{OHOA}$ embedded at the lipid-water interface. Such repulsions would increase the effective headgroup area of the embedded 2OHOA molecules at the lipid-water interface, modifying its spontaneous curvature. Figure 5 summarizes the $\mathrm{pH}$-induced phase transitions and changes in the lattice parameters for all phases detected on increasing $\mathrm{pH}$ from 2.0 to 4.5 for both $2 \mathrm{OHOA} /$ GMO samples prepared at mass ratios of $3 / 7$ and $1 / 1$. The cubic $\operatorname{Im} 3 m$ phases in both 2OHOA/GMO dispersions summarized in Table $S 1$ have smaller lattice parameters at $\mathrm{pH} 3.0$ and 3.5 than pristine GMO cubosomes with $a_{\operatorname{Im} 3 m}=$ $13.1 \mathrm{~nm}$ around the same $\mathrm{pH}$ (see Figure S1). This is consistent with the integration of $2 \mathrm{OHOA}$ into the hydrophobic domains of the structure at this $\mathrm{pH}$ value. On the other hand, upon reaching $\mathrm{pH} 4.0$ and 4.5 in dispersions with $2 \mathrm{OHOA} / \mathrm{GMO}$ mass ratios of $3 / 7$ and $1 / 1$, respectively, the $a_{I m 3 m}$ was found to be greater than that of GMO cubosomes in 


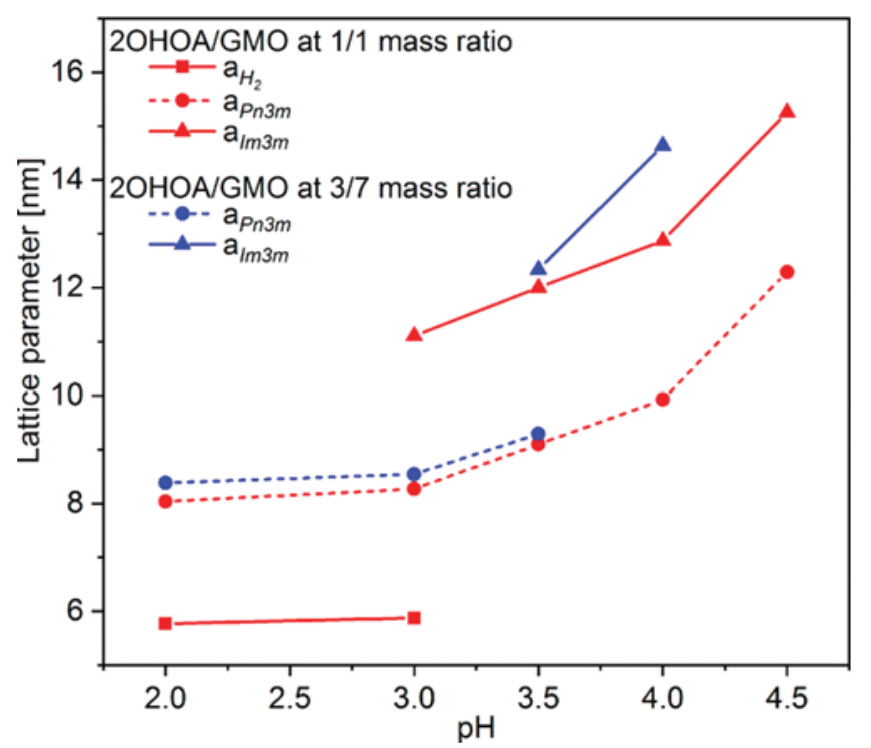

Figure 5. $\mathrm{pH}$-dependent changes in the lattice parameters of the $\mathrm{H}_{2}$ $(\mathbf{\square}), \operatorname{Pn} 3 m(\boldsymbol{\bullet})$, and $\operatorname{Im} 3 m(\boldsymbol{\Delta})$ phases as derived from SAXS patterns of the two samples prepared at $1 / 1$ (red) and $3 / 7$ (blue) 2OHOA/GMO mass ratios presented in Figures 2 and 4. For numerical values see Table S1.

absence of 2OHOA (Figure S1). The later dispersion, measured as control, showed only a slight structural alteration in response to $\mathrm{pH}$ change between 3.0 and 7.0. The increase in $a_{\text {Im } 3 m}$ from $13.1 \mathrm{~nm}$ at $\mathrm{pH} 3.0$ to $13.5 \mathrm{~nm}$ at $\mathrm{pH} 7.0$ may be attributed to the potential presence of trace concentrations of $\mathrm{OA}$ as a hydrolysis product in the sample. ${ }^{68}$ However, this change in lattice parameter in the 2OHOA free cubosomes is insignificant compared to that in the $2 \mathrm{OHOA} / \mathrm{GMO}$ system reported above. The larger $\mathrm{pH}$-induced increase in the lattice parameters of the internal liquid crystalline phases of the 2OHOA/GMO nanocarriers at $\mathrm{pH} 4.0-4.5$, as compared to that at $\mathrm{pH} 3.0-3.5$, reflected a higher degree of $2 \mathrm{OHOA}$ deprotonation and indicated the closer proximity to the apparent $\mathrm{p} K_{\mathrm{a}}\left(\mathrm{p} K_{\mathrm{a}}^{\mathrm{app}}\right)$ of the embedded 2OHOA (Table S1). Upon increasing the $\mathrm{pH}$ to $>5.0$, the 2OHOA/GMO cubosomes eventually transformed into vesicles.

The $\mathrm{pH}$ effect on the $\zeta$-potential of $2 \mathrm{OHOA} / \mathrm{GMO}$ nanoself-assemblies is demonstrated in Figure 6. At $\mathrm{pH} 2.0$, the measurements for the dispersions with $1 / 1$ and $3 / 7$ 2OHOA/ GMO mass ratios indicated the occurrence of rather neutral surfaces with $\zeta$-potential values of $-0.4 \pm 0.7$ and $-3.8 \pm 2.9$ $\mathrm{mV}$, respectively. The $\zeta$-potential of these dispersions was observed to gradually decrease upon increasing $\mathrm{pH}$, with the largest drop in $\zeta$-potential occurring around $\mathrm{pH}$ 6.0, and reaching $-30.9 \pm 4.8$ and $-29.1 \pm 3.5 \mathrm{mV}$ at $\mathrm{pH} 9.0$ for the samples with $1 / 1$ and $3 / 7$ 2OHOA/GMO mass ratios, respectively. This decrease in $\zeta$-potential is mostly caused by the deprotonation of $2 \mathrm{OHOA}$, and correlated well with the discussed nanostructural transitions observed by SAXS and cryo-TEM.

For the system at $2 \mathrm{OHOA} / \mathrm{GMO}$ mass ratio of $1 / 1$, the decrease in the $\zeta$-potential from $-5.8 \pm 2.8$ to $-31.3 \pm 1.7 \mathrm{mV}$ on increasing $\mathrm{pH}$ from 5.0 to $\mathrm{pH} 7.5$ could be associated with an increase in the electrostatic repulsions between the negatively charged 2OHOA/GMO bilayers in the MLVs. This charge repulsion between bilayer sheets could then induce the formation of the swollen MLVs with bilayer-

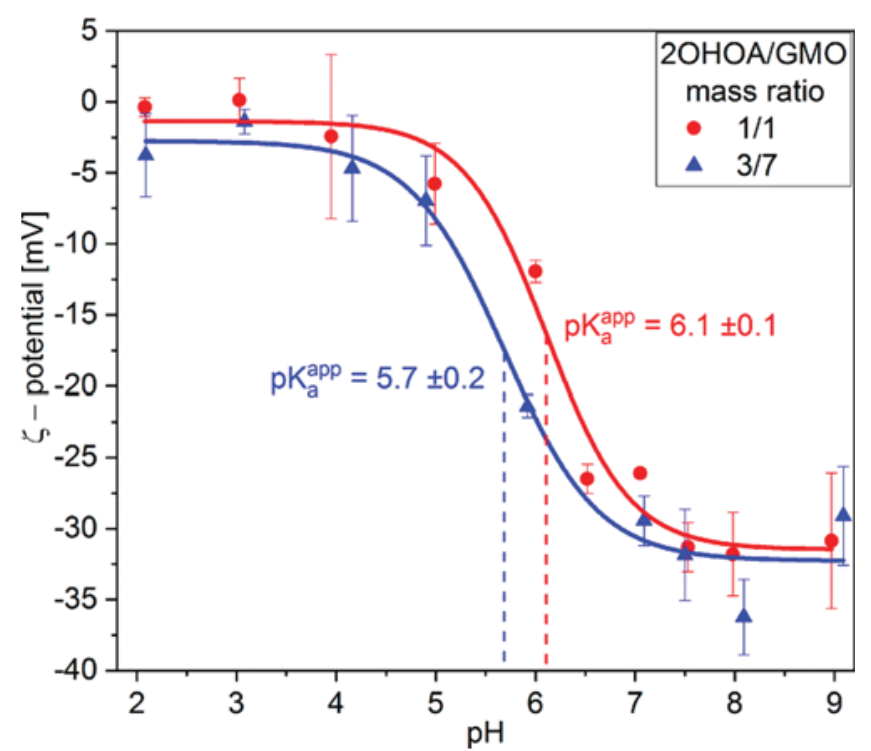

Figure 6. $\mathrm{pH}$-dependent $\zeta$-potential values for the dispersions prepared at $1 / 1$ (red) and 3/7 (blue) 2OHOA/GMO mass ratios. Fits of eq S5 to the data (see SI) are represented as lines and used to estimate the apparent $\mathrm{p} K_{\mathrm{a}}$ of $2 \mathrm{OHOA}$ in the samples prepared at two different lipid compositions.

bilayer distances above $10 \mathrm{~nm}$ at $\mathrm{pH} 6.0$ and 7.4, as reported above.

The $\mathrm{p} K_{\mathrm{a}}^{\text {app }}$ values of $2 \mathrm{OHOA}$ in the nano-self-assemblies at 2OHOA/GMO mass ratios of $1 / 1$ and $3 / 7$ were calculated from the $\mathrm{pH}$-dependent $\zeta$-potential values (see Figure 6 and $\mathrm{SI})$. The resulting $\mathrm{p} K_{\mathrm{a}}^{\mathrm{app}}$ values are $6.1 \pm 0.1$ and $5.7 \pm 0.2$, respectively. These $\mathrm{p} K_{\mathrm{a}}^{\mathrm{app}}$ values are slightly larger than the previously reported $\mathrm{p} K_{\mathrm{a}}^{\mathrm{app}}$ of 5.4 for $2 \mathrm{OHOA}$ in Triton X-100 micelles. ${ }^{64}$ This may be caused by the different 2OHOA content in the $20 \mathrm{HOA} / \mathrm{GMO}$ nano-self-assemblies (2OHOA/lipid mass ratios of $1 / 1$ and $3 / 7$ ) as compared to a mass ratio $1 / 9$ in $2 \mathrm{OHOA} /$ Triton $\mathrm{X}-100$ micelles. The higher concentration of ionizable carboxylic groups on the oilwater interface increases the negative surface-charge density, which could enhance the accumulation of protons, leading to an increase in $\mathrm{p} K_{\mathrm{a}}^{\mathrm{app}} .^{52,72}$ The results suggest that the $\mathrm{p} K_{\mathrm{a}}^{\mathrm{app}}$ of 2OHOA in the designed 2OHOA/GMO nanocarriers may be controlled through changes in the lipid composition. This could potentially be used to tune the phase boundaries of these self-assemblies to desired $\mathrm{pH}$ ranges for specific delivery applications.

The detected $\mathrm{pH}$-triggered structural transformations in this study can be of interest in the development of 2OHOA/GMO nanocarriers for tumor-targeted delivery applications. In the investigated nanocarriers, the major changes in the protonation state of $2 \mathrm{OHOA}$ occurred within the typical $\mathrm{pH}$ range of the extracellular tumor environment of $\mathrm{pH} 5.5-6.5 .^{11,12}$ The modifications of the $2 \mathrm{OHOA} / \mathrm{GMO}$ ratio in these nanocarriers was further found to modulate the $\mathrm{p} K_{\mathrm{a}}^{\mathrm{app}}$ of $2 \mathrm{OHOA}$. Thus, the nanocarriers could be fine-tuned to obtain $\mathrm{pH}$-triggered phase transitions at specific $\mathrm{pH}$ values by optimizing the lipid composition. Additionally, the introduction of hydrophobic additives, such as vitamin $\mathrm{E}$ or triglycerides, could increase the hydrophobic tail volume. ${ }^{73-78}$ This could also shift the phase boundaries between cubosomes and vesicles to higher $\mathrm{pH}$ values, even close to the physiological $\mathrm{pH}$ of 7.4. The reported $\mathrm{pH}$-triggered nanostructural transformations could also trigger 
specific interactions of the nanocarriers with the cells, similar to previous observations on antimicrobial peptide nanocarriers. $^{70}$ This would improve the targeted delivery of this anticancer agent and may focus its exposure to the acidic tumor microenvironment, minimizing off-target side-effects. Further in vitro and in vivo studies are required to determine the practical application of these nano-self-assemblies in cancer therapy.

\section{CONCLUSIONS}

The design and characterization of $\mathrm{pH}$-responsive nanocarriers based on mixtures of $2 \mathrm{OHOA}$ with GMO are presented. In a concentration and $\mathrm{pH}$ dependent manner, 2OHOA was found to actively participate and modulate the structural features of GMO dispersion. The protonation state of the embedded $2 \mathrm{OHOA}$ at the lipid-water interfacial area dictated the structural and morphological characteristics of 2OHOA/ GMO nanocarriers. At $\mathrm{pH}<4.0$, the mostly protonated 2OHOA integrated into the hydrophobic domains of the cubosomes, shrinking their internal channel dimensions and promoting the formation of the $\mathrm{H}_{2}$ phase. However, at $\mathrm{pH}$ $\geq 4.0$, the gradual deprotonation of the $2 \mathrm{OHOA}$ molecules on increasing $\mathrm{pH}$ resulted in electrostatic repulsions among its deprotonated carboxyl-headgroups at the lipid-water interface. This caused the swelling of the cubic $P n 3 m$ and $\operatorname{Im} 3 m$ phases and eventually, at $\mathrm{pH} \geq 4.5$ the colloidal transformation from cubosomes to vesicles.

The results contribute to the fundamental understanding of the self-assembly of $\mathrm{pH}$-responsive and surfactant-like lipids. The presented experimental findings on the designed $\mathrm{pH}$ responsive 2OHOA/GMO nano-self-assemblies could guide the development of advanced cancer nanomedicines for targeted delivery of chemotherapeutics.

\section{ASSOCIATED CONTENT}

\section{Supporting Information}

The Supporting Information is available

Details on the critical packing parameter model, SAXS data analysis, and calculation of the $\mathrm{p} K_{\mathrm{a}}^{\mathrm{app}}$ from the $\zeta$ potential data (PDF)

\section{AUTHOR INFORMATION}

\section{Corresponding Authors}

*E-mail: stefan.salentinig@unifr.ch.

*E-mail: anan.yaghmur@sund.ku.dk.

ORCID

Mark Gontsarik: 0000-0001-7613-5137

Anan Yaghmur: 0000-0003-1608-773X

Stefan Salentinig: 0000-0002-7541-2734

\section{Author Contributions}

"R.P. and M.G. contributed equally to this work.

Notes

The authors declare no competing financial interest.

\section{ACKNOWLEDGMENTS}

Authors are grateful to Heinz Amenitsch and Jianing Li for technical support during synchrotron SAXS experiments and Tillmann Pape (Core Facility for Integrated Microscopy, University of Copenhagen) for technical assistance with cryo-
TEM imaging. The authors acknowledge the Swiss National Science Foundation (Project 200021 169513 held by StS) for funding this research. Financial support to AY by the Danish Council for Independent Research, Technology and Production Sciences, reference $1335-00150 \mathrm{~b}$, is gratefully acknowledged. AY further acknowledges financial support from the Danish Natural Sciences Research Council (DanScatt) for SAXS experiments.

\section{ABBREVIATIONS}

2-OHOA 2-hydroxyoleic acid GMO glycerol monooleate

SAXS small-angle X-ray scattering

cryo-TEM cryogenic-transmission electron microscopy

$\mathrm{H}_{2} \quad$ inverted type hexagonal phase

PBS phosphate buffer saline

MLVs multilamellar vesicles

ULVs unilamellar vesicles

\section{REFERENCES}

(1) Siegel, R. L.; Miller, K. D.; Jemal, A. Cancer Statistics, 2018. CaCancer J. Clin. 2018, 68 (1), 7-30.

(2) Wang, H.; Naghavi, M.; Allen, C.; Barber, R. M.; Carter, A.; Casey, D. C.; Charlson, F. J.; Chen, A. Z.; Coates, M. M.; Coggeshall, M.; et al. Global, Regional, and National Life Expectancy, All-Cause Mortality, and Cause-Specific Mortality for 249 Causes of Death, 1980-2015: A Systematic Analysis for the Global Burden of Disease Study 2015. Lancet 2016, 388, 1459-1544.

(3) Fitzmaurice, C.; Allen, C.; Barber, R. M.; Barregard, L.; Bhutta, Z. A.; Brenner, H.; Dicker, D. J.; Chimed-Orchir, O.; Dandona, R.; Dandona, L.; et al. Global, Regional, and National Cancer Incidence, Mortality, Years of Life Lost, Years Lived with Disability, and Disability-Adjusted Life-Years for 32 Cancer Groups, 1990 to 2015: A Systematic Analysis for the Global Burden of Disease Study. JAMA Oncol. 2017, 3 (4), 524-548.

(4) Terry, A. R.; Plotkin, S. R. Chemotherapy: Present and Future. Otolaryngol. Clin. North Am. 2012, 45 (2), 471-486.

(5) Sawyers, C. Targeted Cancer Therapy. Nature 2004, 432 (7015), 294-297.

(6) Brannon-Peppas, L.; Blanchette, J. O. Nanoparticle and Targeted Systems for Cancer Therapy. Adv. Drug Delivery Rev. 2012, 64, 206212

(7) Peer, D.; Karp, J. M.; Hong, S.; Farokhzad, O. C.; Margalit, R.; Langer, R. Nanocarriers as an Emerging Platform for Cancer Therapy. Nat. Nanotechnol. 2007, 2 (12), 751-760.

(8) Bae, Y. H.; Park, K. Targeted Drug Delivery to Tumors: Myths, Reality and Possibility. J. Controlled Release 2011, 153 (3), 198-205.

(9) Dai, L.; Liu, J.; Luo, Z.; Li, M.; Cai, K. Tumor Therapy: Targeted Drug Delivery Systems. J. Mater. Chem. B 2016, 4 (42), 6758-6772.

(10) Bor, G.; Azmi, I. D. M.; Yaghmur, A. Nanomedicines for Cancer Therapy: Current Status, Challenges and Future Prospects. Ther. Delivery 2019, 10 (2), 113-132.

(11) Tannock, I. F.; Rotin, D. Acid PH in Tumors and Its Potential for Therpeutic Exploitation. Cancer Res. 1989, 49 (16), 4373-4384.

(12) Kato, Y.; Ozawa, S.; Miyamoto, C.; Maehata, Y.; Suzuki, A.; Maeda, T.; Baba, Y. Acidic Extracellular Microenvironment and Cancer. Cancer Cell Int. 2013, 13 (1), 1-8.

(13) Negrini, R.; Fong, W.; Boyd, B. J.; Mezzenga, R. PHResponsive Lyotropic Liquid Crystals and Their Potential Therapeutic Role in Cancer Treatment. Chem. Commun. 2015, 51 (30), $6671-6674$

(14) Chen, W.; Meng, F.; Cheng, R.; Zhong, Z. PH-Sensitive Degradable Polymersomes for Triggered Release of Anticancer Drugs: A Comparative Study with Micelles. J. Controlled Release 2010, 142 (1), $40-46$. 
(15) Sim, T.; Lim, C.; Hoang, N. H.; Oh, K. T. Recent Advance of PH-Sensitive Nanocarriers Targeting Solid Tumors. J. Pharm. Invest. 2017, 47 (5), 383-394.

(16) Maeda, H.; Wu, J.; Sawa, T.; Matsumura, Y.; Hori, K. Tumor Vascular Permeability and the EPR Effect in Macromolecular Therapeutics: A Review. J. Controlled Release 2000, 65 (1), 271-284.

(17) Cammas, S.; Suzuki, K.; Sone, C.; Sakurai, Y.; Kataoka, K.; Okano, T. Thermo-Responsive Polymer Nanoparticles with a CoreShell Micelle Structure as Site-Specific Drug Carriers. J. Controlled Release 1997, 48 (2-3), 157-164.

(18) Soga, O.; Van Nostrum, C. F.; Fens, M.; Rijcken, C. J. F.; Schiffelers, R. M.; Storm, G.; Hennink, W. E. Thermosensitive and Biodegradable Polymeric Micelles for Paclitaxel Delivery. J. Controlled Release 2005, 103 (2), 341-353.

(19) Jang, E. J.; Choi, W. R.; Kim, S. Y.; Hong, S. S.; Rhee, I.; Lee, S. J.; Choi, S. W.; Choi, H. G.; Lim, S. J. 2-Hydroxyoleic Acid-Inserted Liposomes as a Multifunctional Carrier of Anticancer Drugs. Drug Delivery 2017, 24 (1), 1587-1597.

(20) Escribá, P.; Busquets, X.; Inokuchi, J.; Balogh, G.; Török, Z.; Horváth, I.; Harwood, J. L.; Vígh, L. Membrane Lipid Therapy: Modulation of the Cell Membrane Composition and Structure as a Molecular Base for Drug Discovery and New Disease Treatment. Prog. Lipid Res. 2015, 59, 38-53.

(21) Martin, M. L.; Barceló-Coblijn, G.; De Almeida, R. F. M.; Noguera-Salvà, M. A.; Terés, S.; Higuera, M.; Liebisch, G.; Schmitz, G.; Busquets, X.; Escribá, P. V. The Role of Membrane Fatty Acid Remodeling in the Antitumor Mechanism of Action of 2Hydroxyoleic Acid. Biochim. Biophys. Acta, Biomembr. 2013, 1828 (5), 1405-1413.

(22) Teres, S.; Llado, V.; Higuera, M.; Barcelo-Coblijn, G.; Martin, M. L.; Noguera-Salva, M. A.; Marcilla-Etxenike, A.; Garcia-Verdugo, J. M.; Soriano-Navarro, M.; Saus, C.; et al. 2-Hydroxyoleate, a Nontoxic Membrane Binding Anticancer Drug, Induces Glioma Cell Differentiation and Autophagy. Proc. Natl. Acad. Sci. U. S. A. 2012, 109 (22), 8489-8494.

(23) Llado, V.; Gutierrez, A.; Martínez, J.; Casas, J.; Terés, S.; Higuera, M.; Galmés, A.; Saus, C.; Besalduch, J.; Busquets, X.; et al. Minerval Induces Apoptosis in Jurkat and Other Cancer Cells. J. Cell. Mol. Med. 2009, 14 (3), 659-670.

(24) Martinez, J.; Vogler, O.; Casas, J.; Barcelo, F.; Alemany, R.; Prades, J.; Nagy, T.; Baamonde, C.; Kasprzyk, P. G.; Teres, S.; et al. Membrane Structure Modulation, Protein Kinase c Activation, and Anticancer Activity of Minerval. Mol. Pharmacol. 2004, 67 (2), 531540.

(25) Llado, V.; Teres, S.; Higuera, M.; Alvarez, R.; Noguera-Salva, M. A.; Halver, J. E.; Escriba, P. V.; Busquets, X. Pivotal Role of Dihydrofolate Reductase Knockdown in the Anticancer Activity of 2Hydroxyoleic Acid. Proc. Natl. Acad. Sci. U. S. A. 2009, 106 (33), 13754-13758.

(26) Marcilla-Etxenike, A.; Martın, M. L.; Noguera-Salva, M. A.; Garc1, M.; Anto, M.; Busquets, X.; Soriano-navarro, M.; Dey, I.; Escriba, P. V. 2-Hydroxyoleic Acid Induces ER Stress and Autophagy in Various Human Glioma Cell Lines. PLoS One 2012, 7 (10), e48235.

(27) Torgersen, M. L.; Klokk, T. I.; Kavaliauskiene, S.; Klose, C.; Simons, K.; Skotland, T.; Sandvig, K. The Anti-Tumor Drug 2Hydroxyoleic Acid (Minerval) Stimulates Signaling and Retrograde Transport. Oncotarget 2016, 7 (52), 86871-86888.

(28) Chen, Y.; Ma, P.; Gui, S. Cubic and Hexagonal Liquid Crystals as Drug Delivery Systems. BioMed Res. Int. 2014, 2014, 1-12.

(29) Gontsarik, M.; Buhmann, M. T.; Yaghmur, A.; Ren, Q.; Maniura-weber, K.; Salentinig, S. Antimicrobial Peptide-Driven Colloidal Transformations in Liquid- Crystalline Nanocarriers. J. Phys. Chem. Lett. 2016, 7 (17), 3482-3486.

(30) Milak, S.; Zimmer, A. Glycerol Monooleate Liquid Crystalline Phases Used in Drug Delivery Systems. Int. J. Pharm. 2015, 478 (2), 569-587.
(31) Nazaruk, E.; Majkowska-Pilip, A.; Bilewicz, R. Lipidic CubicPhase Nanoparticles-Cubosomes for Efficient Drug Delivery to Cancer Cells. ChemPlusChem 2017, 82 (4), 570-575.

(32) Larsson, K. Cubic Lipid-Water Phases: Structures and Biomembrane Aspects. J. Phys. Chem. 1989, 93 (21), 7304-7314.

(33) Salentinig, S.; Tangso, K. J.; Hawley, A.; Boyd, B. J. PH-Driven Colloidal Transformations Based on the Vasoactive Drug Nicergoline. Langmuir 2014, 30 (49), 14776-14781.

(34) Azmi, I. D. M.; Moghimi, S. M.; Yaghmur, A. Cubosomes and Hexosomes as Versatile Platforms for Drug Delivery. Ther. Delivery 2015, 6 (12), 1347-1364.

(35) Yaghmur, A.; Glatter, O. Characterization and Potential Applications of Nanostructured Aqueous Dispersions. Adv. Colloid Interface Sci. 2009, 147-148, 333-342.

(36) Hyde, S. T. Bicontinuous Structures in Lyotropic Liquid Crystals and Crystalline Hyperbolic Surfaces. Curr. Opin. Solid State Mater. Sci. 1996, 1 (5), 653-662.

(37) Seddon, J. M. Structure of the Inverted Hexagonal (HII) Phase, and Non-Lamellar Phase Transitions of Lipids. Biochim. Biophys. Acta, Rev. Biomembr. 1990, 1031 (1), 1-69.

(38) Guo, C.; Wang, J.; Cao, F.; Lee, R. J.; Zhai, G. Lyotropic Liquid Crystal Systems in Drug Delivery. Drug Discovery Today 2010, 15 (23-24), 1032-1040.

(39) Shao, X.; Bor, G.; Al-Hosayni, S.; Salentinig, S.; Yaghmur, A. Structural Characterization of Self-Assemblies of New Omega-3 Lipids: Docosahexaenoic Acid and Docosapentaenoic Acid Monoglycerides. Phys. Chem. Chem. Phys. 2018, 20, 23928-23941.

(40) Yaghmur, A.; Al-hosayni, S.; Amenitsch, H.; Salentinig, S. Structural Investigation of Bulk and Dispersed Inverse Lyotropic Hexagonal Liquid Crystalline Phases of Eicosapentaenoic Acid Monoglyceride. Langmuir 2017, 33 (49), 14045-14057.

(41) Luzzati, V. The Structure of the Liquid-Crystalline Phases of Lipid-Water Systems. J. Cell Biol. 1962, 12 (2), 207-219.

(42) Barauskas, J.; Johnsson, M.; Tiberg, F. Self-Assembled Lipid Superstructures : Beyond Vesicles and Liposomes. Nano Lett. 2005, 5 (8), 1615-1619.

(43) Kaasgaard, T.; Drummond, C. J. Ordered 2-D and 3-D Nanostructured Amphiphile Self-Assembly Materials Stable in Excess Solvent. Phys. Chem. Chem. Phys. 2006, 8 (43), 4957-4975.

(44) Fong, C.; Le, T.; Drummond, C. J. Lyotropic Liquid Crystal Engineering-ordered Nanostructured Small Molecule Amphiphile Self-Assembly Materials by Design. Chem. Soc. Rev. 2012, 41 (3), $1297-1322$.

(45) Tiberg, F.; Johnsson, M. Drug Delivery Applications of NonLamellar Liquid Crystalline Phases and Nanoparticles. J. Drug Delivery Sci. Technol. 2011, 21 (1), 101-109.

(46) Drummond, C. J.; Fong, C. Surfactant Self-Assembly Objects as Novel Drug Delivery Vehicles. Curr. Opin. Colloid Interface Sci. 1999, 4 (6), 449-456.

(47) Malmsten, M. Phase Transformations in Self-Assembly Systems for Drug Delivery Applications. J. Dispersion Sci. Technol. 2007, 28 (1), 63-72.

(48) Yaghmur, A.; Rappolt, M.; Larsen, S. W. In Situ Forming Drug Delivery Systems Based on Lyotropic Liquid Crystalline Phases: Structural Characterization and Release Properties. J. Drug Delivery Sci. Technol. 2013, 23 (4), 325-332.

(49) Negrini, R.; Mezzenga, R. PH-Responsive Lyotropic Liquid Crystals for Controlled Drug Delivery. Langmuir 2011, 27 (9), 52965303.

(50) Gontsarik, M.; Mohammadtaheri, M.; Yaghmur, A.; Salentinig, S. PH-Triggered Nanostructural Transformations in Antimicrobial Peptide/Oleic Acid Self-Assemblies. Biomater. Sci. 2018, 6 (4), 803812.

(51) Fong, W.; Hanley, T.; Boyd, B. J. Stimuli Responsive Liquid Crystals Provide 'on-Demand' Drug Delivery in Vitro and in Vivo. J. Controlled Release 2009, 135 (3), 218-226.

(52) Salentinig, S.; Sagalowicz, L.; Glatter, O. Self-Assembled Structures and PKa Value of Oleic Acid in Systems of Biological Relevance. Langmuir 2010, 26 (14), 11670-11679. 
(53) Agasti, S. S.; Chompoosor, A.; You, C.; Ghosh, P.; Kim, C. K.; Rotello, V. M. Photoregulated Release of Caged Anticancer Drugs from Gold Nanoparticles to Regulate Drug Release, Minimizing Side Effects and Improving. J. Am. Chem. Soc. 2009, 131, 5728-5729.

(54) Bayer, C. L.; Peppas, N. A. Advances in Recognitive, Conductive and Responsive Delivery Systems. J. Controlled Release 2008, 132 (3), 216-221.

(55) Gao, Z. G.; Fain, H. D.; Rapoport, N. Controlled and Targeted Tumor Chemotherapy by Micellar-Encapsulated Drug and Ultrasound. J. Controlled Release 2005, 102 (1), 203-222.

(56) Barriga, H. M. G.; Holme, M. N.; Stevens, M. M. Cubosomes : The Next Generation of Smart Lipid Nanoparticles? Angew. Chem., Int. Ed. 2019, 58, 2958-2978.

(57) Otte, A.; Soh, B.; Yoon, G.; Park, K. Liquid Crystalline Drug Delivery Vehicles for Oral and IV/Subcutaneous Administration of Poorly Soluble (and Soluble) Drugs. Int. J. Pharm. 2018, 539, 175183.

(58) Nasr, M.; Ghorab, M. K.; Abdelazem, A. In Vitro and in Vivo Evaluation of Cubosomes Containing 5-Fluorouracil for Liver Targeting. Acta Pharm. Sin. B 2015, 5 (1), 79-88.

(59) Jain, V.; Swarnakar, N. K.; Mishra, P. R.; Verma, A.; Kaul, A.; Mishra, A. K.; Jain, N. K. Paclitaxel Loaded PEGylated Gleceryl Monooleate Based Nanoparticulate Carriers in Chemotherapy. Biomaterials 2012, 33 (29), 7206-7220.

(60) Hammersley, A. P.; Svensson, S. O.; Hanfland, M.; Fitch, A. N.; Hausermann, D. Two-Dimensional Detector Software: From Real Detector to Idealised Image or Two-Theta Scan. High Pressure Res. 1996, 14, 235-248.

(61) Smoluchowski, M. Handbuch Der Electrizität Und Des Magnetismus (Graetz). Leipzig, Ger. Barth 1921, 2, 366.

(62) Gustafsson, J.; Ljusberg-wahren, H.; Almgren, M.; Larsson, K. Submicron Particles of Reversed Lipid Phases in Water Stabilized by a Nonionic Amphiphilic Polymer. Langmuir 1997, 13 (26), 69646971.

(63) Landh, T. Phase Behavior in the System Pine Needle Oil Monoglycerides-Poloxamer 407-Water at 20.Degree. J. Phys. Chem. 1994, 98, 8453-8467.

(64) Barceló, F.; Prades, J.; Funari, S. S.; Frau, J.; Alemany, R.; Escribá, P. V. The Hypotensive Drug 2-Hydroxyoleic Acid Modifies the Structural Properties of Model Membranes of Model Membranes. Mol. Membr. Biol. 2004, 21, 261-268.

(65) Hyde, S. T. Microstructure of Bicontinuous Surfactant Aggregates. J. Phys. Chem. 1989, 93, 1458-1464.

(66) Chung, H.; Caffrey, M. The Neutral Area Surface of the Cubic Mesophase: Location and Properties. Biophys. J. 1994, 66, 377-381.

(67) Yaghmur, A.; Laggner, P.; Sartori, B.; Rappolt, M. Calcium Triggered $\mathrm{L} \alpha-\mathrm{H} 2$ Phase Transition Monitored by Combined Rapid Mixing and Time-Resolved Synchrotron SAXS. PLoS One 2008, 3 (4), e2072.

(68) Murgia, S.; Caboi, F.; Monduzzi, M.; Ljusberg-Wahren, H.; Nylander, T. Acyl Migration and Hydrolysis in Monoolein-Based Systems. Springer Berlin Heidelberg 2002, 120, 41-46.

(69) Suga, K.; Kondo, D.; Otsuka, Y.; Okamoto, Y.; Umakoshi, H. Characterization of Aqueous Oleic Acid/Oleate Dispersions by Fluorescent Probes and Raman Spectroscopy. Langmuir 2016, 32, $7606-7612$.

(70) Gontsarik, M.; Yaghmur, A.; Ren, Q.; Maniura-Weber, K.; Salentinig, S. From Structure to Function: PH-Switchable Antimicrobial Nano-Self-Assemblies. ACS Appl. Mater. Interfaces 2019, 11, 2821-2829.

(71) Salentinig, S.; Phan, S.; Darwish, T. A.; Kirby, N.; Boyd, B. J.; Gilbert, E. P. PH-Responsive Micelles Based on Caprylic Acid. Langmuir 2014, 30, 7296-7303.

(72) Cistola, D. P.; Hamilton, J. A.; Jackson, D.; Small, D. M. Ionization and Phase Behavior of Fatty Acids in Water: Application of the Gibbs Phase Rule. Biochemistry 1988, 27 (6), 1881-1888.

(73) Nilsson, C.; Edwards, K.; Eriksson, J.; Larsen, S. W.; Østergaard, J.; Larsen, C.; Urtti, A.; Yaghmur, A. Characterization of Oil-Free and Oil-Loaded Liquid-Crystalline Particles Stabilized by
Negatively Charged Stabilizer Citrem. Langmuir 2012, 28 (32), $11755-11766$.

(74) Yaghmur, A.; De Campo, L.; Sagalowicz, L.; Leser, M. E.; Glatter, O. Emulsified Microemulsions and Oil-Containing Liquid Crystalline Phases. Langmuir 2005, 21 (2), 569-577.

(75) Yaghmur, A.; De Campo, L.; Salentinig, S.; Sagalowicz, L.; Leser, M. E.; Glatter, O. Oil-Loaded Monolinolein-Based Particles with Confined Inverse Discontinuous Cubic Structure $(\mathrm{Fd} 3 \mathrm{~m})$. Langmuir 2006, 22 (2), 517-521.

(76) Yaghmur, A.; Sartori, B.; Rappolt, M. Self-Assembled Nanostructures of Fully Hydrated Monoelaidin- Elaidic Acid and Monoelaidin-Oleic Acid Systems. Langmuir 2012, 28, 10105-10119.

(77) Amar-yuli, I.; Garti, N. Transitions Induced by Solubilized Fat into Reverse Hexagonal Mesophases. Colloids Surf., B 2005, 43, 7282.

(78) Prades, J.; Funari, S. S.; Escribá, P. V.; Barceló, F. Effects of Unsaturated Fatty Acids and Triacylglycerols on Phosphatidylethanolamine Membrane Structure. J. Lipid Res. 2003, 44, 1720-1727. 